\title{
LIE AND JORDAN IDEALS IN PRIME RINGS WITH DERIVATIONS
}

\author{
MANSOOR AHMAD
}

\begin{abstract}
ABSTRACr. In this paper derivations on Lie and Jordan ideals of a prime ring $R$ are studied. The following results are proved. (i) Let $R$ be a prime ring of characteristic not 2, and let $U$ be a Lie or Jordan ideal of $R$. If $d$ is a derivation defined on $U$, and if $a$ is an element of the subring $T(U)$, generated by $U$, or $a$ is an element of $R$, according as $U$ is a Lie or Jordan ideal of $R$, such that $a d u=0$, for all $u \in U$, then either $a=0$ or $d u=0$. (ii) Let $d_{1}, d_{2}$ be derivations defined for all $u \in U$, and also for $u^{2}$ and $u^{3}$ if $U$ is a Lie ideal of $R$, such that the iterate $d_{1} d_{2}$ is also a derivation, satisfying the same conditions as $d_{1}, d_{2}$. Let $d_{1}(u) \in U$, whether $U$ is a Lie or Jordan ideal of $R$. Then, at least, one of $d_{1}(u)$ and $d_{2}(u)$ is zero, for all $u \in U$.
\end{abstract}

Introduction. Lemma 1 of Posner [1] states that if $d$ is a derivation of prime ring $R$ and $a$ an element of $\mathrm{R}$, such that $a d(r)=0$, for all $r \in R$, then either $a=0$ or $d$ is zero. Theorem 1 of Posner [1], which is a direct consequence of Lemma 1, states that if $R$ is a prime ring of characteristic not 2 and if $d_{1}, d_{2}$ are derivations of $R$ such that the iterate $d_{1} d_{2}$ is also a derivation, then at least one of $d_{1}, d_{2}$ is zero. The object of this paper is to generalize these results to Lie and Jordan ideals of $R$.

All rings considered in this paper are associative. For definitions, see [2].

We prove the following results:

LEMMA. Let $R$ be a prime ring of characteristic not 2 and let $U$ be a Lie or Jordan ideal of $R$. If $d$ is a derivation defined on $U$, and if $a$ is an element of the subring $T(U)$, generated by $U$, or a is an element of $R$, according as $U$ is a Lie or Jordan ideal of $R$, such that $a d u=0$, for all $u \in U$, then either $a=0$ or $d u$ $=0$, for all $u \in U$. Further, if $U$ is a Lie ideal of $R$ and if $d(x)$ is defined for all $x \in T(U)$, then at least one of the three statements: $a=0, T(U)$ is in the centre of $R$, and $d(r)=0$ for all $r \in R$, is true. If $U$ is a Jordan ideal of $R$ and if $d(r)$ is defined for all $r \in R$, then either $a=0$ or $d$ is zero.

Proof. Let $U$ be a Lie ideal of $R$. Since $a d u=0$, for all $u \in U$, we have

$$
a d(u r-r u)=0,
$$

for all $u \in U, r \in R$. Putting $r u$ for $r$ in (1) and using (1), we have

$$
a(u r-r u) d u=0
$$

Received by the editors December 2, 1974 and, in revised form, August 1, 1975. AMS (MOS) subject classifications (1970). Primary 16A66, 16A72; Secondary 16A48. Key words and phrases. Prime rings, Lie and Jordan ideals, derivation. 
for all $u \in U, r \in R$. Putting $x a y$ for $r$ in (2) we have

$$
a[(u x a-x a u) y+x a(u y-y u)] d u=0 .
$$

By (2), with $y$ for $r$, the second term vanishes, and so we have $d u=0$ or

$$
a(u x a-x a u)=0
$$

for all $x \in R$, that is

$$
a[(u x-x u) a+x(u a-a u)]=0 .
$$

Putting, $x a$ for $x$ in (4) and using (3), we have

$$
a x a(u a-a u)=0 .
$$

Since $R$ is prime, it follows that either $a=0$ or

$$
a(u a-a u)=0 .
$$

Since $a d v=0, v \in U$, right multiplication of (4) by $d v$ gives

$$
a x(u a-a u) d v=0 .
$$

Since $a d v=0$, and $R$ is prime, we have either $a=0$ or

$$
a u d v=0 .
$$

If $U$ is a Lie ideal of $R$, by hypothesis, $a \in T(U)$, and so $(a x-x a) \in U$, for all $x \in R$. Putting $(a x-x a)$ for $u$ in (6), we have $a^{2} x d v=0$. Since $R$ is prime, either $a^{2}=0$ or $d v=0$, for all $v \in U$. If $a^{2}=0,(5)$ reduces to

$$
a u a=0,
$$

for all $u \in U$. Putting $u x-x u$ for $u$ in (7), $x \in R$, and combining the result thus obtained with (4), we have $a x(u a-a u)=0$, for all $x \in R$. Since $R$ is prime, either $a=0$ or $u a-a u=0$. If $u a-a u=0$, putting $u=a r-r a, r$ $=R$, in this result, we have

$$
a^{2} r+r a^{2}-2 a r a=0,
$$

for all $r \in R$. Since $R$ is not of characteristic 2 , and since $a^{2}=0$, (8) reduces to ara $=0$, for all $r \in R$, and so $a=0$. If $d v=0$, for all $v \in U$, and if $d(x)$ is defined for all $x \in T(U)$, putting $v=x r-r x, x \in T(U), r \in R$, we have

$$
d(x r-r x)=0 .
$$

Putting $r x$ for $r$ in (9) and using (9), we have $(x r-r x) d x=0$, for all $r \in R$, and so, it follows easily that either $T(U)$ is in the centre of $R$ or $d(x)=0$, for all $x \in T(U)$. If the first alternative does not hold, by [2, Theorem 1.2], $T(U)=R$, and so $d(r)$ is defined for all $r \in R$ and $d$ is zero. If $U$ is a Jordan ideal of $R$, and if $R$ is not of characteristic 2, by [2, Theorem 1.1], xcy $\in U$, for all $x, y \in R$, where $c=k b+b k \neq 0, k, b \in U$. Putting $x c y v$ for $u, v$ $\in U$, in $a d u=0$, and using $a d(x c y)=0$, we have axcy $d v=0$. Since $R$ is prime and $c \neq 0$, either $a=0$ or $d v=0$, for all $v \in U$. If $d v=0$ and if $d(r)$ 
is defined for all $r \in R$, then since, by [2, Theorem 1.1], $c r \in U$, for all $r \in R$, putting $v=c r$, and using $d(c)=0$, we have $c d(r)=0$. Since $c \neq 0$, by Lemma 1 of Posner [1], $d$ is zero.

REMARK. Other results are obtained in the case when $R$ is of characteristic 2 .

THEOREM. Let $R$ be a prime ring of characteristic not 2 , and let $d_{1}, d_{2}$ be derivations defined for the elements $u$ of a Lie or Jordan ideal $U$ of $R$, and also for $u^{2}$ and $u^{3}$ if $U$ is a Lie ideal of $R$, such that the iterate $d_{1} d_{2}$ is also a derivation, satisfying the same conditions as $d_{1}, d_{2}$. Let $d_{1}(u) \in U$, for all $u \in U$, whether $U$ is a Lie or Jordan ideal of $R$. Then, at least, one of $d_{1}(u)$ and $d_{2}(u)$ is zero, for all $u \in U$. Further, if $U$ is a Lie ideal of $R$, and if $d(x)$ is defined for all $x, x \in T(U)$, then either $T(U)$ is in the centre of $R$ or at least one of $d_{1}(r)$ and $d_{2}(r)$ is zero, for all $r \in R$. If $U$ is a Jordan ideal of $R$, and if $d(r)$ is defined for all $r \in R$, then at least one of $d_{1}(r)$ and $d_{2}(r)$ is zero, for all $r \in R$.

Proof. Let $d$ denote either of $d_{1}, d_{2}$, and let $U$ be a Lie ideal of $R$. Since, by hypothesis, $d$ is defined for $u$ and $u^{2}, u \in U$, it is defined for $(u+v)^{2}, v \in U$, and so it is defined for $(u v+v u)$ but $(u v-v u) \in U$, therefore it is defined for $(u v-v u)$. Adding and using the fact that $R$ is not of characteristic 2 , it follows that $d$ is defined for $u v$. Also, since by hypothesis $d$ is defined for $u^{3}, u \in U$, it is defined for $(v+u)^{3}+(v-u)^{3}-2 v^{3}$ i.e. for $u^{2} v+u v u+v u^{2}$; but it is defined for $u(u v-v u)-(u v-v u) u$, and so it follows that $d$ is defined for $u v u$ and $u^{2} v+v u^{2}$. Since $u^{2} v-v u^{2} \in U$, it follows that $d$ is defined for $u^{2} v$. Putting $u+w$ for $u, w \in U$, it follows that $d$ is defined for $(u w+w u) v$, but it is defined for $(u w-w u) v$. Therefore, it follows that $d$ is defined for $u v w$. Also, if $k \in U, r \in R$, we have

$$
\begin{aligned}
(u r-r u) v w k & =u r v w k-r u v w k=u r v w k-u v w k r+(u v w k) r-r(u v w k) \\
& =u(r(v w k)-(v w k) r)+(u v w k) r-r(u v w k) .
\end{aligned}
$$

By [2, Theorem 1.3], either $U$ is in the centre of $R$ or $U$ contains every element $x y-y x, x, y \in R$. In each case $r(v w k)-(v w k) r \in U$ and $(u v w k) r-r(u v w k)$ $\in U$. Consequently, by the last identity, it follows that $d$ is defined for $(u r-r u) v w k$. In the same way we can show that it is defined for everyone of the products $u(v r-r v) w k, u v(w r-r w) k$ and $u v w(k r-r k)$.

Now, let $U$ be a Jordan ideal of $R$. By [2, Theorem 1.1], $U$ contains every element $\quad x \alpha, \alpha x, x \alpha y, x, y \in R, \alpha=h k+k h \neq 0, k \in U$. Consequently, any finite product of elements of $R$, at least one of which is $\alpha$, is contained in $U$, and so $d$ is defined for such a product.

If $U$ is a Lie ideal of $R$, we suppose that either each of $a$ and $b$ is an element of $U, a b$ is a product of three elements of $U$, or a product of four elements of $U$, at least one of which is $d_{1}(\beta) r-r d_{1}(\beta), \beta \in U, r \in R$. While, if $U$ is a Jordan ideal of $R$, we choose $b=x \alpha y$, where $x, y$ and $\alpha$ have the same meaning as before. Then, as in the proof of Theorem 1 of Posner [1], it follows that

$$
d_{2}(a) d_{1}(b)+d_{1}(a) d_{2}(b)=0 .
$$

Since, by hypothesis, $d_{1}(c) \in U$, for all $c \in U$, putting $a d_{1}(c)$ for $a$ in this result and using it, we have

$$
d_{2}(a) d_{1}(c) d_{1}(b)+d_{1}(a) d_{1}(c) d_{2}(b)=0 .
$$


But $d_{1}(c) d_{2}(b)=-d_{2}(c) d_{1}(b)$. Therefore, we have

$$
\left(d_{2}(a) d_{1}(c)-d_{1}(a) d_{2}(c)\right) d_{1}(b)=0 .
$$

If $U$ is a Jordan ideal of $R$, we choose $c=r_{1} \alpha r_{2}, r_{1}, r_{2} \in R, \alpha$ being the same as before. Putting $c$ for $b$ in the first result and multiplying the result thus obtained by $d_{1}(b)$ on the right, we have

$$
\left(d_{2}(a) d_{1}(c)+d_{1}(a) d_{2}(c)\right) d_{1}(b)=0 .
$$

Since $R$ is not of characteristic 2 , adding the last two results, we have

$$
d_{2}(a) d_{1}(c) d_{1}(b)=0 .
$$

In view of the first result, this can be put in the form

$$
d_{1}(a) d_{2}(c) d_{1}(b)=0
$$

and then in the form

$$
d_{1}(a) d_{1}(c) d_{2}(b)=0 .
$$

Now, putting $a\left(d_{1}(\beta) r-r d_{1}(\beta)\right), \beta \in U, r \in R$, for $a$ in the last result, according as $U$ is a Lie or Jordan ideal of $R$, and using the last result with $\beta$ for $a$, we have

$$
d_{1}(a) d_{1}(\beta) r d_{1}(c) d_{2}(b)=0
$$

for all $r \in R$. Since $R$ is prime, we have $d_{1}(a) d_{1}(\beta)=0$ or $d_{1}(c) d_{2}(b)=0$. Therefore, if $U$ is a Lie ideal of $R$, by the lemma, it follows that one of $d_{1}(a), d_{1}(\beta), d_{1}(c), d_{2}(b)$ is zero. If $U$ is a Jordan ideal of $R$ and if $d_{1}(a) d_{1}(\beta)$ $=0$, again, by the lemma, it follows that one of $d_{1}(a)$ and $d_{1}(\beta)$ is zero. However, if $U$ is a Jordan ideal of $R$ and if $d_{1}(c) d_{2}(b)=0$, since according to our supposition $b=x \alpha y$, we have $d_{1}(c) d_{2}(x \alpha y)=0$. Putting $r_{1} \alpha r_{2} r_{3}, r_{3}$ $\in R$,for $x$ in this result and using this result, we have $d_{1}(c) r_{1} \alpha r_{2} d_{2}\left(r_{3} \alpha y\right)=0$. Since $R$ prime either $d_{1}(c)=0$ or $d_{2}\left(r_{3} \alpha y\right)=0$. Since, according to our supposition, $c=r_{1} \alpha r_{2}$, we have $d\left(r_{1} \alpha r_{2}\right)=0$, where $d$ denotes $d_{1}$ or $d_{2}$. Putting $u r_{1}$ for $r_{1}, u \in U$, in this result and using this result, we have $d(u) r_{1} \alpha r_{2}$. Since $R$ is prime and $x \neq 0$, we have $d(u)=0$, for all $u \in U$.

The proof of the second part of the theorem is the same as that of the second part of the lemma.

ACKNOWLEDGEMENT. The author wishes to thank the referee for helpful comments and remarks.

\section{REFERENCES}

1. E. C. Posner, Derivations in prime rings, Proc. Amer. Math. Soc. 8 (1957), 1093-1100.MR 20 \#2361.

2. I. N. Herstein, Topics in ring theory, Univ. of Chicago Press, Chicago, Ill., 1969. MR 42 \#6018. 